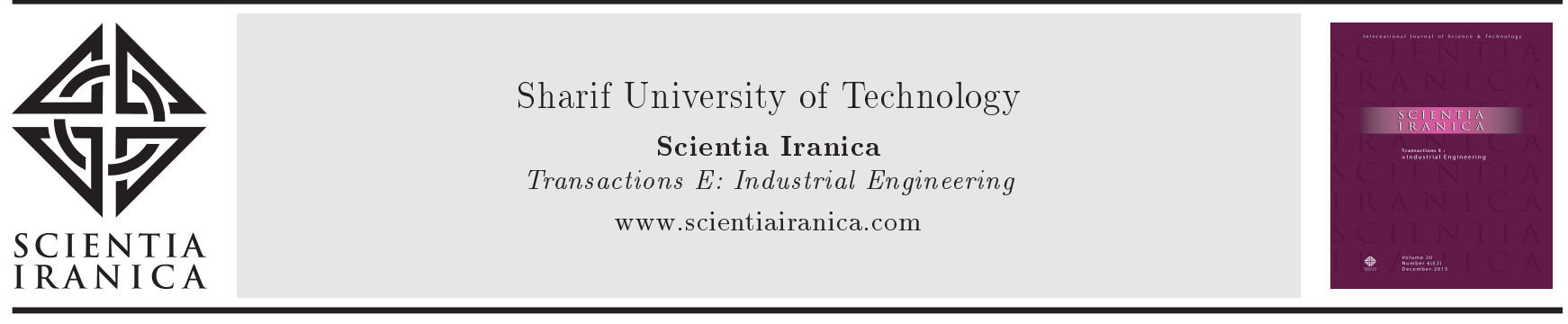

\title{
A distance-based decision model in interval-valued hesitant fuzzy setting for industrial selection problems
}

\author{
H. Gitinavard ${ }^{a}$, S.M. Mousavi ${ }^{\mathrm{b}}$, B. Vahdani ${ }^{\mathrm{c}}$ and A. Siadat ${ }^{\mathrm{d}, *}$ \\ a. School of Industrial Engineering, Iran University of Science and Technology, Tehran, Iran. \\ b. Department of Industrial Engineering, Faculty of Engineering, Shahed University, Tehran, Iran. \\ c. Faculty of Industrial and Mechanical Engineering, Qazvin Branch, Islamic Azad University, Qazvin, Iran. \\ d. Laboratoire de Conception, Fabrication Commande, Arts et Métier Paris Tech. Centre de Metz, Metz, France. \\ Received 21 November 2014; received in revised form 5 June 2015; accepted 10 August 2015
}

KEYWORDS
Distance-based
decision model;
Interval-valued
hesitant fuzzy sets;
Multi-criteria decision
making;
Group decision
making;
Industrial robot
selection problems.

\section{Introduction}

An important part in operational research and modern decision science is Multi-Criteria Decision Making (MCDM), which includes multiple decision alternatives and multiple decision criteria. The MCDM methods try to find the most suitable potential alternative(s) from a set of candidate alternatives versus the conflicting criteria $[1,2]$. The methods are applied to

*. Corresponding author. Tel.: +33785122844 E-mail addresses: ali.siadat@ensam.eu (A. Siadat); sm.mousavi@shahed.ac.ir (S.M. Mousavi)

\begin{abstract}
In a classical Group Decision-Making (GDM) analysis, the ratings of potential alternatives and the weights of criteria or Decision Makers (DMs) are known precisely. However, for dealing with uncertain situations, the DMs can define their opinions in inguistic variables based on fuzzy sets in industrial selection problems. In this respect, an Interval-Valued Hesitant Fuzzy Set (IVHFS) is the suitable and capable theory that could help the DMs with assigning some interval-valued membership degrees to a candidate option under a set. This paper introduces a novel Interval-Valued Hesitant Fuzzy Distance-Based Group Decision (IVHF-DBGD) model by a group of DMs, in which the best potential alternative can be appraised and selected among the conflicting criteria. n the proposed IVHF-DBGD model, the weight of each criterion is determined by extended IVHF-entropy method along with the DMs' opinions about the criteria's weights. Also, the weight of each DM is computed by a new IVHF-order preference method with the relative closeness. Moreover, this paper introduces a new IVHF-collective index to discriminate among potential alternatives in the selection process. Finally, the computational results with a robot selection from the literature indicate that the proposed IVHF-DBGD model is the suitable group decision-making tool for the industrial selection problems.
\end{abstract}

(C) 2016 Sharif University of Technology. All rights reserved. 
MCDM. In addition, by the two presented methods, they ranked the high risks in BOT projects as fuzzy techniques for order preference by similarity to ideal solution (FTOPSIS) and fuzzy linear programming technique for multidimensional analysis of preference (FLINMAP) method. Vahdani et al. [4] developed a compromise solution based on the concept that the most suitable potential alternative should be close to a positive ideal solution and further from the negative ideal solution concurrently.

In addition, a group of experts or Decision Makers (DMs) is established for dealing with the complexity of the engineering and of environmental management, and discriminating all relevant aspects of decision-making problems. On the other hand, MCDM methods by a group of experts; namely MCGDM, consider the DMs' judgments and preferences to consist of quantitative and/or qualitative ratings of criteria as well as criteria's weights. In the recent decade, some studies have focused on MCGDM problems to demonstrate reliable results in the real-life situations [7-10].

Zhang and $\mathrm{Xu}$ [11] studied about the uncertain linguistic information aggregation, which led to extending the ordered weighted averaging and continuous ordered weighted geometric operators. $\mathrm{Xu}$ [9] introduced some operations and relations in interval-valued intuitionistic fuzzy numbers, and described some matrices as interval-valued intuitionistic fuzzy similarity and equivalence matrix; then, by regarding their properties, a distance-based method was extended for group decision analysis under interval-valued intuitionistic fuzzy matrices. Yue [7] developed TOPSIS method for computing the weight of each DM by interval fuzzy number for group decision problems. Yu and Lai [8] proposed a distance-based methodology under a group decision-making analysis to solve the emergency problems. Chen [10] extended a signed-distance-based method to specify the relative importance of criteria. In the proposed method, the interval type- 2 trapezoidal fuzzy number was utilized to indicate the ratings of the potential alternatives and the relative importance of different criteria.

Some authors have studied on the industrial selection problems, such as the robot selection problem. An industrial robot is utilized in a widely diverse area such as move tools, parts, materials, and other devices regarding the reprogrammable multi-functional manipulator [12]. To address the issues, Agrawal et al. [13] proposed a procedure to rank and select the best robot by applying the TOPSIS method. In addition, an expert system was developed to help the DMs to construct the visualizations and priorities of the robot selection problem process. Goh et al. [14] extended a revised weighted sum decision model by regarding both the subjective and objective criteria to choose the desirable robot for an industrial selection problem. Parkan and Wu [15] presented the interrelationship and applications of the TOPSIS and operational competitiveness rating methods in the industrial robot selection problem. Then, the results of the proposed methods were compared with other approaches. Chu and Lin [16] focused on a fuzzy TOPSIS method for solving the industrial robot selection problem. In addition, the ratings of potential alternatives regarding the various subjective criteria and criteria's weights were expressed by fuzzy numbers. Bhattacharya et al. [17] integrated Quality Function Deployment (QFD) and AHP methods by considering the four candidate robots and seven technical requirements. Kahraman et al. [18] extended a hierarchical TOPSIS method under uncertainty to select the most suitable robot. Kumar and Garg [19] proposed a deterministic quantitative model with distance-based mechanism for assessing, ranking, and selecting the best robot. Kentli and Kar [20] applied a distance measurement technique and satisfaction function to solve the industrial robot selection problems.

The Interval-Valued Hesitant Fuzzy Sets (IVHFSs) theory can be a very powerful tool to deal with uncertain and imprecise information for the industrial robot selection problems. The IVHFS theory has been first introduced by Chen et al. [21] that could help the DMs assign their opinions to an option under a set of margins of errors. In this paper, a novel Interval-Valued Hesitant Fuzzy Distance-Based Group Decision (IVHF-DBGD) model by a group of experts is presented for industrial selection problems, such as evaluating and ranking the best industrial robot. The main advantages and merits of the proposed IVHF-DBGD model are provided as follows: (1) introducing a new interval-valued hesitant fuzzy collective index in the ranking process to discriminate among potential alternatives; (2) proposing a new interval-valued hesitant fuzzy order preference method with the relative closeness for determining the weight of each DM; (3) extending an interval-valued hesitant fuzzy entropy method along with considering the DMs' opinions for obtaining the weight of each criterion; and (4) considering the DMs' preference judgments on the performance rating of the alternatives and the relative importance of criteria with linguistic variables by taking IVHFSs theory.

The structure of this paper is arranged as follows: In Section 2, some relations as well as operations are reviewed under the interval-valued hesitant fuzzy environment. In Section 3, the proposed IVHF-DBGD model is illustrated. A practical example for the robot selection problem is presented to indicate the validity and capability of the proposed model in Section 4 . Finally, in Section 5, we end the paper with some conclusions and recommendations. 


\section{Basic concepts and operations}

In this section, we review some basic preliminaries under interval-valued hesitant fuzzy environment that are utilized in the proposed IVHF-DBGD model.

Definition 1 [21]. Let $X$ be a universe set, then the HIVFS on $X$ is represented as follows:

$$
\tilde{E}=\left\{\left\langle x_{i}, \tilde{h}_{E}\left(x_{i}\right)\right\rangle \mid x_{i} \in X, i=1,2, \ldots, n\right\}
$$

where $\tilde{h}_{E}\left(x_{i}\right)$ is the interval membership degree for an element of $x_{i} \in X$ under set $E$. In addition, the $\tilde{h}_{E}\left(x_{i}\right)$ is defined as a Hesitant Interval-Valued Fuzzy Element (HIVFE) that satisfies the following relation:

$$
\tilde{h}_{E}\left(x_{i}\right)=\left\{\tilde{\gamma} \mid \tilde{\gamma} \in \tilde{h}_{\tilde{E}}\left(x_{i}\right)\right\}
$$

where the interval number is $\tilde{\gamma}=\left[\tilde{\gamma}^{L}, \tilde{\gamma}^{U}\right] ; \tilde{\gamma}^{L}$ and $\tilde{\gamma}^{U}$ are defined as the lower and upper bounds of $\tilde{\gamma}$ respectively.

Definition 2 [21]. Consider $\tilde{h}, \tilde{h}_{1}$ and $\tilde{h}_{2}$ as three HIVFE, then the basic operations are defined as below:

$$
\begin{gathered}
\tilde{h}^{\lambda}=\left\{\left[\left(\tilde{\gamma}^{L}\right)^{\lambda},\left(\tilde{\gamma}^{U}\right)^{\lambda}\right] \mid \tilde{\gamma} \in \tilde{h}\right\}, \quad \lambda>0, \\
\lambda \tilde{h}=\left\{\left[1-\left(1-\tilde{\gamma}^{L}\right)^{\lambda}, 1-\left(1-\tilde{\gamma}^{U}\right)^{\lambda}\right] \mid \tilde{\gamma} \in \tilde{h}\right\}, \\
\lambda>0, \\
\tilde{h}_{1} \oplus \tilde{h}_{2}=\left\{\left[\gamma_{1}^{L}+\gamma_{2}^{L}-\gamma_{1}^{L} \gamma_{2}^{L}, \gamma_{1}^{U}+\gamma_{2}^{U}-\gamma_{1}^{U} \gamma_{2}^{U}\right]\right. \\
\tilde{h}_{1} \otimes \tilde{h}_{2}=\left\{\left[\tilde{\gamma}_{1}^{L} \in \tilde{h}_{1}, \tilde{\gamma}_{2} \in \tilde{h}_{2}\right\},\right. \\
\left.\left.\gamma_{1}^{U} \gamma_{2}^{U}\right] \mid \tilde{\gamma}_{1} \in \tilde{h}_{1}, \tilde{\gamma}_{2} \in \tilde{h}_{2}\right\} .
\end{gathered}
$$

Definition 3. Consider $E=\left\{h_{1}, h_{2}, \ldots, h_{n}\right\}$ as a collection of HIVFEs, then Eqs. (5) and (6) are extended based on Definition 2:

$$
\begin{gathered}
\oplus_{i=1}^{n} h_{i}=\underset{\gamma_{1} \in h_{1}, \gamma_{1} \in h_{2}, \ldots, \gamma_{n} \in h_{n}}{ }\left\{\left[1-\Pi_{i=1}^{n}\left(1-\gamma_{i}^{L}\right),\right.\right. \\
\left.\left.1-\Pi_{i=1}^{n}\left(1-\gamma_{i}^{U}\right)\right]\right\}, \\
h_{1} \otimes h_{2} \otimes \ldots \otimes h_{n}=\bigcup_{\gamma_{1} \in h_{1}, \gamma_{2} \in h_{2}, \ldots, \gamma_{n} \in h_{n}}\left\{\left[\Pi_{i=1}^{n} \gamma_{i}^{L},\right.\right. \\
\left.\left.\Pi_{i=1}^{n} \gamma_{i}^{U}\right]\right\} .
\end{gathered}
$$

Definition 4 [22]. The subtraction and division relations for the HFS are defined as follows:

$$
\begin{gathered}
h_{1}-h_{2}=\bigcup_{\gamma_{1} \in h_{1}, \gamma_{2} \in h_{2}}\left\{\begin{array}{cc}
\frac{\gamma_{1}-\gamma_{2}}{1-\gamma_{2}} & \text { if } \gamma_{1} \geq \gamma_{2} \text { and } \gamma_{2} \neq 1 ; \\
0 & \text { otherwise }
\end{array}\right\}, \\
\frac{h_{1}}{h_{2}}=\bigcup_{\gamma_{1} \in h_{1}, \gamma_{2} \in h_{2}}\left\{\begin{array}{cc}
\frac{\gamma_{1}}{\gamma_{2}} & \text { if } \gamma_{1} \leq \gamma_{2} \text { and } \gamma_{2} \neq 0 ; \\
1 & \text { otherwise }
\end{array}\right\} .
\end{gathered}
$$

Definition 5 [21]. The Hamming and Euclidean distance measures are defined for the HIVFS as follows:

$$
\begin{aligned}
d_{\text {hivh }}\left(h_{M}, h_{N}\right)= & \frac{1}{2 l} \sum_{x_{i}} \sum_{j=1}^{l_{x_{i}}}\left(\left|h_{M}^{\sigma(j) L}\left(x_{i}\right)-h_{N}^{\sigma(j) L}\left(x_{i}\right)\right|\right. \\
& \left.+\left|h_{M}^{\sigma(j) U}\left(x_{i}\right)-h_{N}^{\sigma(j) U}\left(x_{i}\right)\right|\right)
\end{aligned}
$$

where $h_{M}$ and $h_{N}$ are expressed as $h_{\tilde{M}}^{\sigma(j)}\left(x_{i}\right)=$ $\left[h_{\tilde{M}}^{\sigma(j) L}\left(x_{i}\right), h_{\tilde{M}}^{\sigma(j) U}\left(x_{i}\right)\right]$ and $h_{\tilde{N}}^{\sigma(j)}\left(x_{i}\right)=\left[h_{\tilde{N}}^{\sigma(j) L}\left(x_{i}\right)\right.$, $\left.h_{\tilde{N}}^{\sigma(j) U}\left(x_{i}\right)\right]$, respectively, and the $j$ th largest values in $h_{M}$ and $h_{N}$ are represented as $h_{M}^{\sigma(j)}$ and $h_{M}^{\sigma(j)}$ :

$d(\tilde{\alpha}, \tilde{\beta})=\sqrt{\frac{1}{2 l} \sum_{j=1}^{l}\left(\left|\tilde{\alpha}_{\sigma(j)}^{L}-\tilde{\beta}_{\sigma(j)}^{L}\right|^{2}+\left|\tilde{\alpha}_{\sigma(j)}^{U}-\tilde{\beta}_{\sigma(j)}^{U}\right|^{2}\right)}$

where $\tilde{\alpha}$ and $\tilde{\beta}$ are HIVFSs, and the $j$ th largest values in $\tilde{\alpha}^{L}, \tilde{\alpha}^{U}, \tilde{\beta}^{L}$, and $\tilde{\beta}^{U}$ are indicated by $\tilde{\alpha}_{\sigma(j)}^{L}, \tilde{\beta}_{\sigma(j)}^{L}$, $\tilde{\alpha}_{\sigma(j)}^{U}$, and $\tilde{\beta}_{\sigma(j)}^{U}$.

Definition 6 [21]. The Hesitant Interval-Valued Fuzzy Averaging (HIVFA) and Hesitant IntervalValued Fuzzy Weighted Averaging (HIVFWA) relations are defined as follows:

$$
\begin{aligned}
& \operatorname{HIVFA}\left(\tilde{h}_{1}, \tilde{h_{2}}, \ldots, \tilde{h_{n}}\right)=\left(\oplus_{j=1}^{n}\left(\frac{1}{n} \tilde{h}_{j}\right)\right) \\
& =\bigcup_{\tilde{\gamma}_{1} \in \tilde{h}_{1}, \tilde{\gamma}_{2} \in \tilde{h}_{2}, \ldots, \tilde{\gamma}_{n} \in \tilde{h}_{n}}\left\{\left[1-\Pi_{j=1}^{n}\left(1-\gamma_{j}^{L}\right)^{\frac{1}{n}},\right.\right. \\
& \left.\left.1-\Pi_{j=1}^{n}\left(1-\gamma_{j}^{U}\right)^{\frac{1}{n}}\right]\right\}, \\
& \operatorname{HIVFWA}\left(\tilde{h}_{1}, \tilde{h_{2}}, \ldots, \tilde{h_{n}}\right)=\left(\oplus_{j=1}^{n}\left(w_{j} \tilde{h}_{j}\right)\right) \\
& =\bigcup_{\tilde{\gamma}_{1} \in \tilde{h}_{1}, \tilde{\gamma}_{2} \in \tilde{h}_{2}, \ldots, \tilde{\gamma}_{n} \in \tilde{h}_{n}}\left\{\left[1-\Pi_{j=1}^{n}\left(1-\gamma_{j}^{L}\right)^{w_{j}},\right.\right. \\
& \left.\left.1-\Pi_{j=1}^{n}\left(1-\gamma_{j}^{U}\right)^{w_{j}}\right]\right\} \text {, }
\end{aligned}
$$


where $w=\left(w_{1}, w_{2}, \ldots, w_{n}\right)^{T}$ is the weight vector of $\tilde{h}_{j}(j=1,2, \ldots, n)$, and $w_{j}>0$, where $\sum_{j=1}^{n} w_{j}=1$.

Definition 7 [23]. The normalized interval-valued hesitant fuzzy decision matrix $\left(B=\left(b_{i j}\right)_{m \times n}\right)$ is defined as follows:

$$
\begin{aligned}
& b_{i j}=\bigcup_{t_{i j} \in b_{i j}}= \\
& \left\{\begin{array}{lr}
\left\{\left[\gamma_{i j}^{l}, \gamma_{i j}^{u}\right]\right\} & \text { for possitive criteria } \\
\left\{\left[1-\gamma_{i j}^{u}, 1-\gamma_{i j}^{l}\right]\right\} & \text { for negative criteria }
\end{array}\right.
\end{aligned}
$$

\section{Proposed interval-valued hesitant fuzzy distance-based group decision model}

- Step 1. Establish an Interval-Valued Hesitant Fuzzy decision matrix (IVHF-decision matrix) by considering the opinions of a committee of the DMs $(k=1,2, \ldots, K)$ as calculated by Eq. (16), shown in Box I.

- Step 2. Determine the weight of criteria according to the proposed Interval-Valued Hesitant Fuzzy entropy (IVHF-entropy) method.
- Step 2.1. Aggregate the DMs' opinions $\left(\tilde{h}_{p k}\right)$ and establish the IVHF-decision matrix based on Definition 6 as shown in Box II.

- Step 2.2. Aggregate the DMs' opinions for the weight of criteria $\left(\tilde{h}_{w c k}\right)$ as follows:

$$
\begin{aligned}
\nu_{j} & =\operatorname{HIVFA}\left(\tilde{h}_{w c 1}, \tilde{h}_{w c 2}, \ldots, \tilde{h}_{w c k}\right)=\left(\oplus_{k=1}^{K}\left(\frac{1}{k} \tilde{h}_{e c k}\right)\right) \\
& =\bigcup_{\gamma_{w c 1} \in \tilde{h}_{w c 1}, \tilde{\gamma}_{w c 2} \in \tilde{h}_{w c 2}, \ldots, \tilde{\gamma}_{w c k} \in \tilde{h}_{w c k}} \\
& \left\{\left[1-\Pi_{k=1}^{K}\left(1-\gamma_{w c k}^{L}\right)^{\frac{1}{k}},\right.\right. \\
& \left.\left.1-\Pi_{k=1}^{K}\left(1-\gamma_{w c k}^{U}\right)^{\frac{1}{k}}\right]\right\} .
\end{aligned}
$$

- Step 2.3. Specify the $T_{i j}=\left[T_{i j}^{l}, T_{i j}^{u}\right]$ as follows:

$$
T_{i j}^{l}\left\{\begin{array}{lll}
\frac{\mu_{i j}^{l}}{1-\Pi_{i=1}^{m}\left(1-\mu_{i j}^{l}\right)} & \text { if } \mu_{i j}^{l} \leq 1-\Pi_{i=1}^{m}\left(1-\mu_{i j}^{l}\right) \\
& \text { and } 1-\Pi_{i=1}^{m}\left(1-\mu_{i j}^{l}\right) \neq 0 \\
& \text { otherwise }
\end{array}\right.
$$

$$
M=\begin{gathered}
A_{1} \\
\vdots \\
A_{m}
\end{gathered}\left[\begin{array}{ccc}
C_{1} & \cdots & C_{n} \\
\left.\left\{\mu_{11}^{L 1}, \mu_{11}^{U 1}\right],\left[\mu_{11}^{L 2}, \mu_{11}^{U 2}\right], \ldots,\left[\mu_{11}^{L k}, \mu_{11}^{U k}\right]\right\} & \cdots & \left\{\left[\mu_{1 n}^{L 1}, \mu_{1 n}^{U 1}\right],\left[\mu_{1 n}^{L 2}, \mu_{1 n}^{U 2}\right], \ldots,\left[\mu_{1 n}^{L k}, \mu_{1 n}^{U k}\right]\right\} \\
\vdots & \ddots & \vdots \\
\left\{\left[\mu_{m 1}^{L 1}, \mu_{m 1}^{U 1}\right],\left[\mu_{m 1}^{L 2}, \mu_{m 1}^{U 2}\right], \ldots,\left[\mu_{m 1}^{L k}, \mu_{m 1}^{U k}\right]\right\} & \cdots & \left.\left\{\left[\mu_{m n}^{L 1}, \mu_{m n}^{U 1}\right],\left[\mu_{m n}^{L 2}, \mu_{m n}^{U 2}\right], \ldots,\left[\mu_{m n}^{L k}, \mu_{m n}^{U k}\right]\right\}\right]_{m \times n}
\end{array}\right.
$$

Box I

$$
\begin{aligned}
{\left[\mu_{i j}^{L}, \mu_{i j}^{U}\right] } & =\operatorname{HIVFA}\left(\tilde{h}_{p}, \tilde{h}_{p 2}, \ldots, \tilde{h}_{p k}\right) \\
& =\left(\oplus_{k=1}^{K}\left(\frac{1}{k} \tilde{h}_{p k}\right)\right) \\
& =\bigcup_{\tilde{\gamma}_{p 1} \in \tilde{h}_{p 1}, \tilde{\gamma}_{p 2} \in \tilde{h}_{p 2}, \ldots, \tilde{\gamma}_{p k} \in \tilde{h}_{p k}}\left\{\left[1-\Pi_{k=1}^{K}\left(1-\gamma_{p k}^{L}\right)^{\frac{1}{k}}, 1-\Pi_{k=1}^{K}\left(1-\gamma_{p k}^{U}\right)^{\frac{1}{k}}\right]\right\} \\
& A_{1}\left(\begin{array}{cccc}
C_{1} & C_{2} & \ldots & C_{n} \\
{\left[\mu_{11}^{L k}, \mu_{11}^{U k}\right]} & {\left[\mu_{12}^{L k}, \mu_{12}^{U k}\right]} & \cdots & {\left[\mu_{1 n}^{L k}, \mu_{1 n}^{U k}\right]} \\
\vdots & \vdots & \ddots & \vdots \\
{\left[\mu_{m 1}^{L k}, \mu_{m 1}^{U k}\right]} & {\left[\mu_{m 2}^{L k}, \mu_{m 2}^{U k}\right]} & \cdots & {\left[\mu_{m n}^{L k}, \mu_{m n}^{U k}\right]}
\end{array}\right)_{m \times n}
\end{aligned}
$$




$$
T_{i j}^{u} \begin{cases}\frac{\mu_{i j}^{u}}{1-\Pi_{i=1}^{m}\left(1-\mu_{i j}^{u}\right)} & \text { if } \mu_{i j}^{u} \leq 1-\Pi_{i=1}^{m}\left(1-\mu_{i j}^{u}\right) \\ & \text { and } 1-\Pi_{i=1}^{m}\left(1-\mu_{i j}^{u}\right) \neq 0 \\ 1 & \text { otherwise }\end{cases}
$$

- Step 2.4. Estimate the unreliability/degree of deviation of each criterion according to interval-valued hesitant fuzzy entropy matrix $\left(T_{i j}=\left[T_{i j}^{l}, T_{i j}^{u}\right]\right)$ as follows:

$$
\begin{aligned}
& d_{j}=\left[\left(\Pi_{i=1}^{m}\left(1-T_{i j}^{l}\right)^{\operatorname{Ln}\left(T_{i j}^{l}\right)}\right)^{-\frac{1}{\operatorname{Ln}(m)}},\right. \\
& \left.\left(\Pi_{i=1}^{m}\left(1-T_{i j}^{u}\right)^{L n\left(T_{i j}^{u}\right)}\right)^{-\frac{1}{L n(m)}}\right] .
\end{aligned}
$$

- Step 2.5. Specify the final weight of criteria by regarding DMs' judgments.

$$
w_{j}=\frac{\nu_{j}^{l} \cdot d_{j}^{l}\left(\sum_{j=1}^{n} \nu_{j}^{u} \cdot d_{j}^{u}\right)+\nu_{j}^{u} \cdot d_{j}^{u}\left(\sum_{j=1}^{n} \nu_{j}^{l} \cdot d_{j}^{l}\right)}{2\left(\sum_{j=1}^{n} \nu_{j}^{l} \cdot d_{j}^{l}\right)\left(\sum_{j=1}^{n} \nu_{j}^{u} \cdot d_{j}^{u}\right)} \forall j .
$$

- Step 3. Specify the weight of each DM according to the proposed new interval-valued hesitant fuzzy order preference method by the following steps:

- Step 3.1. Construct the weighted normalized IVHF-decision matrix for each DM based on Definition 7 as shown in Box III.
- Step 3.2. Construct the Interval-Valued Hesitant Fuzzy Positive Ideal Solution (IVHF-PIS) and the Interval-Valued Hesitant Fuzzy Negative Ideal Solution (IVHF-NIS) matrixes by Eqs. (25) and (26) as shown in Box IV, where the average of group decision matrix is calculated as follows:

$$
\begin{aligned}
& \mu_{i j}^{* L}=\frac{1}{K} \sum_{k=1}^{K} \mu_{i j}^{L k}, \\
& \mu_{i j}^{* U}=\frac{1}{K} \sum_{k=1}^{K} \mu_{i j}^{U k}, \\
& \mu_{i j}^{-L}=\min _{k}\left\{\mu_{i j}^{L k}\right\}, \\
& \mu_{i j}^{-U}=\max _{k}\left\{\mu_{i j}^{U k}\right\} .
\end{aligned}
$$

- Step 3.3. Calculate a hesitant fuzzy average of group score value $\left(S_{i}\right)$ and a hesitant fuzzy worst group score value $\left(R_{i}\right)$ for each alternative $\left(A_{i}\right)$ as shown in Box V.

- Step 3.4. Specify the relative closeness of each DM by the following relation:

$$
\eta_{k}=\frac{R_{k}}{R_{k}+S_{k}} \forall k
$$

- Step 3.5. Specify each DM's weight with regard to their relative closeness.

$$
\theta_{k}=\frac{\eta_{k}}{\sum_{k=1}^{K} \eta_{k}} \forall k
$$

$$
F^{k}=\left[F_{i j}^{L k}, F_{i j}^{U k}\right]_{m \times n}=\left(\begin{array}{cccc}
w_{1} \cdot\left[h_{11}^{L}, h_{11}^{U}\right] & \ldots & w_{(n-1)} \cdot\left[h_{1(n-1)}^{L}, h_{1(n-1)}^{U}\right] & w_{n} \cdot\left[h_{1 n}^{L}, h_{1 n}^{U}\right] \\
w_{1} \cdot\left[h_{21}^{L}, h_{21}^{U}\right] & \ldots & w_{(n-1) \cdot} \cdot\left[h_{2(n-1)}^{L}, h_{2(n-1)}^{U}\right] & w_{n} \cdot\left[h_{2 n}^{L}, h_{m n}^{U}\right] \\
\vdots & \ddots & \vdots \\
w_{1} \cdot\left[h_{m 1}^{L}, h_{m 1}^{U}\right] & \ldots & w_{(n-1)} \cdot\left[h_{m(n-1)}^{L}, h_{m(n-1)}^{U}\right] & w_{n} \cdot\left[h_{m n}^{L}, h_{m n}^{U}\right]
\end{array}\right)
$$



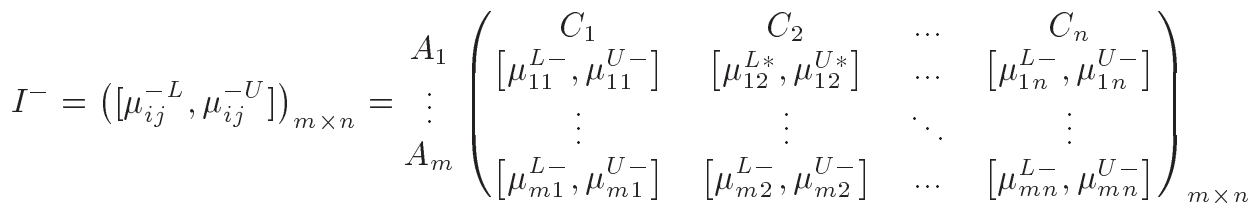




$$
\begin{aligned}
S_{k} & =\sqrt{\frac{1}{2 l_{x_{i}}} \sum_{i=1}^{m} \sum_{j=1}^{n} \sum_{\lambda=1}^{l_{x_{i}}}\left(\left|F_{i j}^{L k \sigma(\lambda)}\left(x_{i}\right)-I_{i j}^{* L \sigma(\lambda)}\left(x_{i}\right)\right|^{2}+\left|F_{i j}^{U \sigma(\lambda)}\left(x_{i}\right)-I_{i j}^{* U \sigma(\lambda)}\left(x_{i}\right)\right|^{2}\right) \forall k} \\
R_{k} & =\sqrt{\frac{1}{2 l_{x_{i}}} \sum_{i=1}^{m} \sum_{j=1}^{n} \sum_{\lambda=1}^{l_{x_{i}}}\left(\left|F_{i j}^{L \sigma(\lambda)}\left(x_{i}\right)-I_{i j}^{-L \sigma(\lambda)}\left(x_{i}\right)\right|^{2}+\left|F_{i j}^{U \sigma(\lambda)}\left(x_{i}\right)-I_{i j}^{-U \sigma(\lambda)}\left(x_{i}\right)\right|^{2}\right) \forall k}
\end{aligned}
$$

here, $w_{j}$ is the final weight of criteria.

Box V

- Step 4. Specify the IVHF-PIS and IVHF-NIS based on Step 3.1, i.e. the weighted normalized IVHF-decision matrix for each DM, by utilizing the following relations:

$$
\begin{aligned}
& A_{j}^{*}=\left\{h_{1}^{*}, h_{2}^{*}, \ldots, h_{n}^{*}\right\}, \\
& h_{j}^{*}= \begin{cases}\left\{x_{j}, \max _{i}\left\langle h_{i j}^{\sigma(\lambda)}\right\rangle\right\}, & \forall J \\
\left.x_{j}, \min _{i}\left\langle h_{i j}^{\sigma(\lambda)}\right\rangle\right\}, & \forall J^{\prime}\end{cases} \\
& A_{j}^{-}=\left\{h_{1}^{-}, h_{2}^{-}, \ldots, h_{n}^{-}\right\}, \\
& h_{j}^{-}= \begin{cases}\left\{x_{j}, \min _{i}\left\langle h_{i j}^{\sigma(\lambda)}\right\rangle\right\}, & \forall J \\
\left\{x_{j}, \max _{i}\left\langle h_{i j}^{\sigma(\lambda)}\right\rangle\right\}, & \forall J^{\prime}\end{cases}
\end{aligned}
$$

where $J$ and $J^{\prime}$ are defined as two sets including benefit criteria and cost criteria, respectively.

- Step 5. Determine the separation measure by calculating the distance values between the weighted normalized IVHF-decision matrix and IVHF-PIS/IVHF-NIS respecting each DM as shown in Box VI.

- Step 6. Compute the proposed collective index $\left(C I_{i}\right)$ for each alternative as follows:

$$
\zeta_{i}=\left(1-\prod_{k=1}^{K}\left(1-\frac{d_{i}^{k *}}{d_{i}^{k-}}\right)\right)^{\frac{1}{K}}+\underset{\forall d_{i}^{k *} \geq d_{i}^{k-}}{\left(1+\partial_{i}\right)} \forall i
$$

$$
\begin{gathered}
\partial_{i}=\left(\max _{k \in A}\left(\frac{d_{i}^{k^{*}}}{d_{i}^{k-}}\right)\right)^{\frac{1}{\max _{j} w_{j}}} \forall\left(d_{i}^{k *} \leq d_{i}^{k-}\right) \in A, i \\
\zeta_{i}=\left(1-\Pi_{k=1}^{K}\left(1-d_{i}^{k *}\right)\right)^{\frac{1}{m}}+\left(1-\Pi_{k=1}^{K}\left(d_{i}^{k-}\right)\right)^{\frac{1}{K}} \forall i \\
C I_{i}=\zeta_{i}+\xi_{i} \forall i
\end{gathered}
$$

- Step 7. Rank the alternatives with regard to the minimum value of collective index $\left(C I_{i}\right)$.

\section{Practical example for ind ustrial robot selection problems}

The practical example from Liang and Wang [24] is presented to indicate the suitability and feasibility of the proposed IVHF-DBGD method. In this illustrative example, we suppose a manufacturing company that needs a robot to implement the materials handling tasks. Three robots $\left(R_{1}, R_{2}\right.$, and $\left.R_{3}\right)$ are the candidates for the appraisement. Four DMs as a committee $\left(\mathrm{DM}_{k}, k=1,2, \ldots, 4\right)$ are considered to evaluate the candidate's alternatives among the conflicting criteria in interval-valued hesitant fuzzy setting. Also, the sixth criteria $\left(C_{j}, j=1,2, \ldots, 6\right)$ has been used as follows:

- Man-machine interface $\left(C_{1}\right)$;

- Programming flexibility $\left(C_{2}\right)$;

$$
\begin{aligned}
& d_{i}^{k^{*}}=\theta_{k} \sum_{j=1}^{n} \sqrt{\frac{1}{2 l_{x_{i}}} \sum_{\lambda=1}^{l_{x i}}\left(\left|F_{i j}^{L \sigma(\lambda)}\left(x_{i}\right)-A_{j}^{* L \sigma(\lambda)}\left(x_{i}\right)\right|^{2}+\left|F_{i j}^{U \sigma(\lambda)}\left(x_{i}\right)-A_{j}^{* U \sigma(\lambda)}\left(x_{i}\right)\right|^{2}\right)} \forall i, k \\
& d_{i}^{k^{-}}=\theta_{k} \sum_{j=1}^{n} \sqrt{\frac{1}{2 l_{x_{i}}} \sum_{\lambda=1}^{l_{x i}}\left(\left|F_{i j}^{L \sigma(\lambda)}\left(x_{i}\right)-A_{j}^{-L \sigma(\lambda)}\left(x_{i}\right)\right|^{2}+\left|F_{i j}^{U \sigma(\lambda)}\left(x_{i}\right)-A_{j}^{-U \sigma(\lambda)}\left(x_{i}\right)\right|^{2}\right)} \forall i, k
\end{aligned}
$$


Table 1. Hesitant linguistic variables for rating the importance of criteria and the decision makers.

\begin{tabular}{lc}
\hline $\begin{array}{c}\text { Hesitant linguistic } \\
\text { variables }\end{array}$ & $\begin{array}{c}\text { Hesitant interval-valued } \\
\text { fuzzy elements }\end{array}$ \\
\hline Very High $(\mathrm{VH})$ & {$[0.90,0.90]$} \\
High $(\mathrm{H})$ & {$[0.75,0.80]$} \\
Medium $(\mathrm{M})$ & {$[0.50,0.55]$} \\
Low $(\mathrm{L})$ & {$[0.35,0.40]$} \\
Very Low $(\mathrm{VL})$ & {$[0.10,0.10]$} \\
\hline
\end{tabular}

Table 2. Hesitant linguistic variables for rating the possible alternatives.

\begin{tabular}{lc}
\hline $\begin{array}{c}\text { Hesitant linguistic } \\
\text { variables }\end{array}$ & $\begin{array}{c}\text { Hesitant interval-valued } \\
\text { fuzzy elements }\end{array}$ \\
\hline Extremely Good (EG) & {$[1.00,1.00]$} \\
Very Very Good (VVG) & {$[0.90,0.90]$} \\
Very Good (VG) & {$[0.80,0.90]$} \\
Good (G) & {$[0.70,0.80]$} \\
Moderately Good (MG) & {$[0.60,0.70]$} \\
Fair (F) & {$[0.50,0.60]$} \\
Moderately Poor (MP) & {$[0.40,0.50]$} \\
Poor (P) & {$[0.25,0.40]$} \\
Very Poor (VP) & {$[0.10,0.25]$} \\
Very Very Poor (VVP) & {$[0.10,0.10]$} \\
\hline
\end{tabular}

- Vendor's service contract $\left(C_{3}\right)$;

- Load capacity $\left(C_{4}\right)$;

- Positioning accuracy $\left(C_{5}\right)$;

- Purchase cost $\left(C_{6}\right)$.

Tables 1 and 2 indicate the HIVFEs for each hesitant fuzzy linguistic variable to determine the relative importance of each criteria/DM and the rating of each possible alternative among the conflicting criteria, respectively. In this case, the linguistic variables are adopted from Vahdani et al. study [25] for rating the alternatives and criteria's weights. In their study, the intuitionistic fuzzy set was utilized to solve the MCGDM problems. Also, as presented in Torra's study [26], the intuitionistic fuzzy sets can be converted to hesitant fuzzy sets. The weight of each criterion is evaluated by utilizing the linguistic variables as follows: Very Low (VL), Low (L), Medium (M), High $(\mathrm{H})$, and Very High $(\mathrm{VH})$. In addition, the ratings of alternatives are assessed by the following linguistic variables: Very Very Poor (VVP), Very Poor (VP), Poor (P), Moderately Poor (MP), Fair (F), Moerately Good (MG), Good (G), Very Good (VG), Very Very Good (VVG), and Extremely Good (EG). Regarding the upper and lower bounds of some linguistic variables (e.g., VH, VL/EG, VG and VVP), we can utilize the expertise of the DMs to determine them.
In addition, as represented in Table 3 , the IVHFdecision matrix is constructed by DMs' judgments under the hesitant fuzzy environment. Thus, the IVHF-decision matrix regarding IVHFEs is established in Table 4. Also, the mentioned process has been considered for criteria's weights. The results are obtained in Tables 5 and 6 .

The weight of each criterion is obtained by the proposed IVHF-entropy method with regard to DMs' opinions about the relative importance of the criteria. As indicated in Table 7, we aggregate the opinions of the DMs, and then specify $T_{i j}$ matrix; their computations are provided by Eqs. (45)-(47):

$$
\begin{aligned}
& {\left[\mu_{11}^{L}, \mu_{11}^{U}\right]=\operatorname{HIVFA}\left(\tilde{h}_{p 1}, \tilde{h}_{p 2}, \tilde{h}_{p 3}, \tilde{h}_{p 4}\right)} \\
& =\left(\oplus_{k=1}^{4}\left(\frac{1}{4} \tilde{h}_{p k}\right)\right) \\
& =\bigcup_{\tilde{\gamma}_{p 1} \in \tilde{h}_{p 1}, \tilde{\gamma}_{p 2} \in \tilde{h}_{p 2}, \tilde{\gamma}_{p 3} \in \tilde{h}_{p 3}, \tilde{\gamma}_{p 4} \in \tilde{h}_{p 4}} \\
& \left\{\left[1-\Pi_{k=1}^{4}\left(1-\gamma_{p k}^{K}\right)^{\frac{1}{4}}, 1-\Pi_{k=1}^{4}\left(1-\gamma_{p k}^{U}\right)^{\frac{1}{4}}\right]\right\} \\
& =\left[1-\left((1-0.5)^{\frac{1}{4}}\right)\left((1-0.5)^{\frac{1}{4}}\right)\right. \\
& \left((1-0.7)^{\frac{1}{4}}\right)\left((1-0.8)^{\frac{1}{4}}\right) \text {, } \\
& 1-\left((1-0.6)^{\frac{1}{4}}\right)\left((1-0.6)^{\frac{1}{4}}\right)\left((1-0.8)^{\frac{1}{4}}\right) \\
& \left.\left((1-0.9)^{\frac{1}{4}}\right)\right]=[0.650036,0.762158] \text {, } \\
& T_{11}^{l}= \begin{cases}\frac{\mu_{11}^{l}}{1-\Pi_{i=1}^{3}\left(1-\mu_{i 1}^{l}\right)} & \text { if } \mu_{11}^{l} \leq 1-\Pi_{i=1}^{3}\left(1-\mu_{i 1}^{l}\right) \\
& \text { and } 1-\Pi_{i=1}^{3}\left(1-\mu_{i 1}^{l}\right) \neq 0 \\
1 & \text { otherwise }\end{cases} \\
& =\frac{0.650036}{1-(1-0.650036)(1-0.559944)(1-0.691992)} \\
& =0.682405 \text {, } \\
& T_{11}^{u}= \begin{cases}\frac{\mu_{11}^{u}}{1-\Pi_{i=1}^{3}\left(1-\mu_{i 1}^{u}\right)} & \text { if } \mu_{11}^{u} \leq 1-\Pi_{i=1}^{3}\left(1-\mu_{i 1}^{u}\right) \\
& \text { and } 1-\Pi_{i=1}^{3}\left(1-\mu_{i 1}^{u}\right) \neq 0 \\
1 & \text { otherwise }\end{cases} \\
& =\frac{0.762158}{1-(1-0.762158)(1-0.663641)(1-0.800000)} \\
& =0.774551 \text {. }
\end{aligned}
$$


Table 3. Performance ratings of the alternatives in hesitant fuzzy linguistic variables.

\begin{tabular}{|c|c|c|c|c|c|}
\hline \multirow{2}{*}{ Criteria } & \multirow{2}{*}{ Alternatives } & \multicolumn{4}{|c|}{ Decision makers } \\
\hline & & $\mathrm{DM}_{1}$ & $\mathrm{DM}_{2}$ & $\mathrm{DM}_{3}$ & $\mathrm{DM}_{4}$ \\
\hline \multirow{3}{*}{$C_{1}$} & $\mathrm{R} 1$ & $\mathrm{~F}$ & $\mathrm{~F}$ & G & $\mathrm{VG}$ \\
\hline & $\mathrm{R} 2$ & $\mathrm{~F}$ & G & $\mathrm{F}$ & $\mathrm{F}$ \\
\hline & $\mathrm{R} 3$ & G & $\mathrm{F}$ & VG & G \\
\hline \multirow{3}{*}{$C_{2}$} & $\mathrm{R} 1$ & G & $\mathrm{P}$ & $\mathrm{G}$ & $\mathrm{F}$ \\
\hline & $\mathrm{R} 2$ & VG & G & VG & $\mathrm{F}$ \\
\hline & $\mathrm{R} 3$ & G & $\mathrm{F}$ & VG & G \\
\hline \multirow{3}{*}{$C_{3}$} & $\mathrm{R} 1$ & $\mathrm{~F}$ & F & G & $\mathrm{F}$ \\
\hline & $\mathrm{R} 2$ & G & $\mathrm{F}$ & VG & G \\
\hline & $\mathrm{R} 3$ & G & G & G & VG \\
\hline \multirow{3}{*}{$C_{4}$} & $\mathrm{R} 1$ & G & $\mathrm{MG}$ & $\mathrm{F}$ & $\mathrm{F}$ \\
\hline & $\mathrm{R} 2$ & MG & G & MG & MG \\
\hline & $\mathrm{R} 3$ & $\mathrm{~F}$ & $\mathrm{~F}$ & G & G \\
\hline \multirow{3}{*}{$C_{5}$} & $\mathrm{R} 1$ & $\mathrm{~F}$ & $\mathrm{P}$ & MP & $\mathrm{F}$ \\
\hline & $\mathrm{R} 2$ & MG & $\mathrm{F}$ & $\mathrm{F}$ & MG \\
\hline & R3 & G & G & MG & G \\
\hline \multirow{3}{*}{$C_{6}$} & $\mathrm{R} 1$ & G & G & G & MG \\
\hline & $\mathrm{R} 2$ & MG & MG & $\mathrm{F}$ & $\mathrm{F}$ \\
\hline & R3 & $\mathrm{F}$ & $\mathrm{F}$ & $\mathrm{P}$ & MP \\
\hline
\end{tabular}

Table 5. DMs' judgments about criteria weights by hesitant fuzzy linguistic variables.

\begin{tabular}{ccccc}
\hline \multirow{2}{*}{ Criteria } & \multicolumn{4}{c}{ Decision } \\
\cline { 2 - 5 } & $\mathbf{D M}_{\mathbf{1}}$ & $\mathbf{D M}_{\mathbf{2}}$ & $\mathbf{D M}_{\mathbf{3}}$ & $\mathbf{D M}_{\mathbf{4}}$ \\
\hline$C_{1}$ & $\mathrm{H}$ & $\mathrm{VH}$ & $\mathrm{VH}$ & $\mathrm{H}$ \\
$C_{2}$ & $\mathrm{VH}$ & $\mathrm{H}$ & $\mathrm{VH}$ & $\mathrm{M}$ \\
$C_{3}$ & $\mathrm{M}$ & $\mathrm{L}$ & $\mathrm{M}$ & $\mathrm{L}$ \\
$C_{4}$ & $\mathrm{VH}$ & $\mathrm{VH}$ & $\mathrm{H}$ & $\mathrm{VH}$ \\
$C_{5}$ & $\mathrm{VH}$ & $\mathrm{H}$ & $\mathrm{H}$ & $\mathrm{H}$ \\
$C_{6}$ & $\mathrm{M}$ & $\mathrm{M}$ & $\mathrm{M}$ & $\mathrm{L}$ \\
\hline
\end{tabular}

In addition, we specify the degree of deviation/ unreliability for each criterion by utilizing Eq. (22). Thus, the criteria's weights are determined by Eq. (23). The results have been shown in Table 8 . Hence, the computations of aggregation and the degree of deviation/unreliability are obtained by Eqs. (48) and (49).

$$
\begin{aligned}
d_{1}= & {\left[\left(\Pi_{i=1}^{3}\left(1-T_{i 1}^{l}\right)^{\operatorname{Ln}\left(T_{i 1}^{l}\right)}\right)^{-\frac{1}{\operatorname{Ln}(3)}},\right.} \\
& \left.\left(\Pi_{i=1}^{3}\left(1-T_{i 1}^{u}\right)^{\operatorname{Ln}\left(T_{i 1}^{u}\right)}\right)^{-\frac{1}{\operatorname{Ln}(3)}}\right] \\
= & {\left[\left(((1-0.682405))^{\operatorname{Ln}(0.682405)}\right)\right.}
\end{aligned}
$$

\begin{tabular}{|c|c|c|c|c|c|}
\hline \multirow[t]{2}{*}{ Criteria } & \multirow[t]{2}{*}{ Alternatives } & \multicolumn{4}{|c|}{ Decision makers } \\
\hline & & $\mathrm{DM}_{1}$ & $\mathrm{DM}_{2}$ & $\mathrm{DM}_{3}$ & $\mathrm{DM}_{4}$ \\
\hline \multirow{3}{*}{$C_{1}$} & $R_{1}$ & {$[0.50,0.60]$} & {$[0.50,0.60]$} & {$[0.70,0.80]$} & {$[0.80,0.90]$} \\
\hline & $R_{2}$ & {$[0.50,0.60]$} & {$[0.70,0.80]$} & {$[0.50,0.60]$} & {$[0.50,0.60]$} \\
\hline & $R_{3}$ & {$[0.70,0.80]$} & {$[0.50,0.60]$} & {$[0.80,0.90]$} & {$[0.70,0.80]$} \\
\hline \multirow{3}{*}{$C_{2}$} & $R_{1}$ & {$[0.70,0.80]$} & {$[0.25,0.40]$} & {$[0.70,0.80]$} & {$[0.50,0.60]$} \\
\hline & $R_{2}$ & {$[0.80,0.90]$} & {$[0.70,0.80]$} & {$[0.80,0.90]$} & {$[0.50,0.60]$} \\
\hline & $R_{3}$ & {$[0.70,0.80]$} & {$[0.50,0.60]$} & {$[0.80,0.90]$} & {$[0.70,0.80]$} \\
\hline \multirow{3}{*}{$C_{3}$} & $R_{1}$ & {$[0.50,0.60]$} & {$[0.50,0.60]$} & {$[0.70,0.80]$} & {$[0.50,0.60]$} \\
\hline & $R_{2}$ & {$[0.70,0.80]$} & {$[0.50,0.60]$} & {$[0.80,0.90]$} & {$[0.70,0.80]$} \\
\hline & $R_{3}$ & {$[0.70,0.80]$} & {$[0.70,0.80]$} & {$[0.70,0.80]$} & {$[0.80,0.90]$} \\
\hline \multirow{3}{*}{$C_{4}$} & $R_{1}$ & {$[0.70,0.80]$} & {$[0.60,0.70]$} & {$[0.50,0.60]$} & {$[0.50,0.60]$} \\
\hline & $R_{2}$ & {$[0.60,0.70]$} & {$[0.70,0.80]$} & {$[0.60,0.70]$} & {$[0.60,0.70]$} \\
\hline & $R_{3}$ & {$[0.50,0.60]$} & {$[0.50,0.60]$} & {$[0.70,0.80]$} & {$[0.70,0.80]$} \\
\hline \multirow{3}{*}{$C_{5}$} & $R_{1}$ & {$[0.50,0.60]$} & {$[0.25,0.40]$} & {$[0.40,0.50]$} & {$[0.50,0.60]$} \\
\hline & $R_{2}$ & {$[0.60,0.70]$} & {$[0.50,0.60]$} & {$[0.50,0.60]$} & {$[0.60,0.70]$} \\
\hline & $R_{3}$ & {$[0.70,0.80]$} & {$[0.70,0.80]$} & {$[0.60,0.70]$} & {$[0.70,0.80]$} \\
\hline \multirow{3}{*}{$C_{6}$} & $R_{1}$ & {$[0.70,0.80]$} & {$[0.70,0.80]$} & {$[0.70,0.80]$} & {$[0.60,0.70]$} \\
\hline & $R_{2}$ & {$[0.60,0.70]$} & {$[0.60,0.70]$} & {$[0.50,0.60]$} & {$[0.50,0.60]$} \\
\hline & $R_{3}$ & {$[0.50,0.60]$} & {$[0.50,0.60]$} & {$[0.25,0.40]$} & {$[0.40,0.50]$} \\
\hline
\end{tabular}

Table 4. Performance ratings of the alternatives in IVHFEs. 
Table 6. DMs' judgments about criteria weights by IVHFEs.

\begin{tabular}{ccccc}
\hline \multirow{2}{*}{ Criteria } & \multicolumn{4}{c}{ Decision makers } \\
\cline { 2 - 5 } & $\mathbf{D M}_{\mathbf{1}}$ & $\mathbf{D M}_{\mathbf{2}}$ & $\mathbf{D M}$ & $\mathbf{D M}_{\mathbf{3}}$ \\
\hline$C_{1}$ & {$[0.75,0.80]$} & {$[0.90,0.90]$} & {$[0.90,0.90]$} & {$[0.75,0.80]$} \\
$C_{2}$ & {$[0.90,0.90]$} & {$[0.75,0.80]$} & {$[0.90,0.90]$} & {$[0.50,0.55]$} \\
$C_{3}$ & {$[0.50,0.55]$} & {$[0.35,0.40]$} & {$[0.50,0.55]$} & {$[0.35,0.40]$} \\
$C_{4}$ & {$[0.90,0.90]$} & {$[0.90,0.90]$} & {$[0.75,0.80]$} & {$[0.90,0.90]$} \\
$C_{5}$ & {$[0.90,0.90]$} & {$[0.75,0.80]$} & {$[0.75,0.80]$} & {$[0.75,0.80]$} \\
$C_{6}$ & {$[0.50,0.55]$} & {$[0.50,0.55]$} & {$[0.50,0.55]$} & {$[0.35,0.40]$} \\
\hline
\end{tabular}

Table 7. Aggregated hesitant fuzzy decision matrix and established $T_{i j}$ matrix.

\begin{tabular}{cccc}
\hline \multicolumn{4}{c}{ Aggregated hesitant fuzzy decision matrix } \\
\hline \multicolumn{1}{c}{$\boldsymbol{R}_{\mathbf{1}}$} & $\boldsymbol{R}_{\mathbf{2}}$ & $\boldsymbol{R}_{\mathbf{3}}$ \\
\hline$C_{1}$ & {$[0.650036,0.762158]$} & {$[0.559944,0.663641]$} & {$[0.691992,0.800000]$} \\
$C_{2}$ & {$[0.571383,0.686983]$} & {$[0.721684,0.831820]$} & {$[0.691992,0.800000]$} \\
$C_{3}$ & {$[0.559944,0.663641]$} & {$[0.691992,0.800000]$} & {$[0.728919,0.831829]$} \\
$C_{4}$ & {$[0.583829,0.686983]$} & {$[0.627758,0.728919]$} & {$[0.612701,0.717157]$} \\
$C_{5}$ & {$[0.420853,0.531930]$} & {$[0.552786,0.653589]$} & {$[0.677629,0.778663]$} \\
$C_{6}$ & {$[0.677629,0.778663]$} & {$[0.552786,0.653589]$} & {$[0.420853,0.531930]$} \\
\hline
\end{tabular}

The constructed $T_{i j}$ matrix

\begin{tabular}{cccc}
\cline { 2 - 4 } & $\boldsymbol{R}_{\mathbf{1}}$ & $\boldsymbol{R}_{\mathbf{2}}$ & $\boldsymbol{R}_{\mathbf{3}}$ \\
\hline$C_{1}$ & {$[0.682405,0.774551]$} & {$[0.587827,0.674432]$} & {$[0.726451,0.813008]$} \\
$C_{2}$ & {$[0.593178,0.694293]$} & {$[0.749212,0.840671]$} & {$[0.718388,0.808512]$} \\
$C_{3}$ & {$[0.581302,0.671235]$} & {$[0.718388,0.809154]$} & {$[0.756723,0.841339]$} \\
$C_{4}$ & {$[0.621086,0.703876]$} & {$[0.667827,0.746843]$} & {$[0.651810,0.734792]$} \\
$C_{5}$ & {$[0.459194,0.551731]$} & {$[0.603145,0.677919]$} & {$[0.739361,0.807648]$} \\
$C_{6}$ & {$[0.739361,0.807648]$} & {$[0.603145,0.677919]$} & {$[0.459194,0.551731]$} \\
\hline
\end{tabular}

$$
\begin{aligned}
& \left((1-0.587827)^{\operatorname{Ln}(0.587827)}\right) \\
& \left.\left((1-0.726451)^{\operatorname{Ln}(0.726451)}\right)\right)^{-\frac{1}{\operatorname{Ln}(3)}}, \\
& \left(\left((1-0.774551)^{\operatorname{Ln}(0.774551)}\right)\right. \\
& \left((1-0.674432)^{\operatorname{Ln}(0.674432)}\right) \\
& \left.\left.\left((1-0.813008)^{\operatorname{Ln}(0.813008)}\right)\right)^{-\frac{1}{\operatorname{Ln}(3)}}\right] \\
& =[0.299786,0.344836]
\end{aligned}
$$

$$
\begin{gathered}
v_{1}=\operatorname{HIVFA}\left(\tilde{h}_{w c 1}, \tilde{h}_{w c 2}, \tilde{h}_{w c 3}, \tilde{h}_{w c 4}\right)=\left(\oplus_{k=1}^{4}\left(\frac{1}{4} \tilde{h}_{w c k}\right)\right) \\
=\bigcup_{\tilde{\gamma}_{w c 1} \in \tilde{h}_{w c 1}, \tilde{\gamma}_{w c 2} \in \tilde{h}_{w c 2}, \tilde{\gamma}_{w c 3} \in \tilde{h}_{w c 3}, \tilde{\gamma}_{w c 4} \in \tilde{h}_{w c 4}}
\end{gathered}
$$

$$
\begin{aligned}
& \left\{\left[1-\Pi_{k=1}^{4}\left(1-\gamma_{w c k}^{L}\right)^{\frac{1}{4}}, 1-\Pi_{k=1}^{4}\left(1-\gamma_{w c k}^{U}\right)^{\frac{1}{4}}\right]\right\} \\
& =\left[1-\left((1-0.75)^{\frac{1}{4}}\right)\left((1-0.9)^{\frac{1}{4}}\right)\left((1-0.9)^{\frac{1}{4}}\right)\right. \\
& \left((1-0.75)^{\frac{1}{4}}\right), 1-\left((1-0.8)^{\frac{1}{4}}\right)\left((1-0.9)^{\frac{1}{4}}\right) \\
& \left.\left((1-0.9)^{\frac{1}{4}}\right)\left((1-0.8)^{\frac{1}{4}}\right)\right] \\
& =[0.841886,0.858578] .
\end{aligned}
$$

The proposed new interval-valued hesitant fuzzy order preference method with the relative closeness is considered to specify the weight of each DM. In this respect, the hesitant fuzzy average of group score value and that of the hesitant fuzzy worst group score value are computed. Then, the relative closeness and the final weight of each DM are determined. The computations are demonstrated by Eqs. (50)(53), and the obtained results are presented in Table 9 . 
Table 8. Unreliability $\left(d_{j}\right)$, aggregated DMs' judgments $\left(\nu_{j}\right)$, and final criteria's weights $\left(w_{j}\right)$.

\begin{tabular}{cccccc}
\hline & $\boldsymbol{d}_{\boldsymbol{j}}$ & & $\boldsymbol{\nu}_{\boldsymbol{j}}$ & & $\boldsymbol{w}_{\boldsymbol{j}}$ \\
\hline$d_{1}$ & {$[0.299786,0.344836]$} & $\nu_{1}$ & {$[0.841886,0.858578]$} & $w_{1}$ & 0.199817 \\
$d_{2}$ & {$[0.309579,0.366568]$} & $\nu_{2}$ & {$[0.811969,0.826794]$} & $w_{2}$ & 0.201772 \\
$d_{3}$ & {$[0.310343,0.363343]$} & $\nu_{3}$ & {$[0.429912,0.480384]$} & $w_{3}$ & 0.111707 \\
$d_{4}$ & {$[0.290328,0.324234]$} & $\nu_{4}$ & {$[0.874256,0.881079]$} & $w_{4}$ & 0.196884 \\
$d_{5}$ & {$[0.292183,0.314821]$} & $\nu_{5}$ & {$[0.801182,0.831820]$} & $w_{5}$ & 0.181043 \\
$d_{6}$ & {$[0.292183,0.314821]$} & $\nu_{6}$ & {$[0.466105,0.516443]$} & $w_{6}$ & 0.108774 \\
\hline
\end{tabular}

Table 9. Results for specifying the DMs' weights.

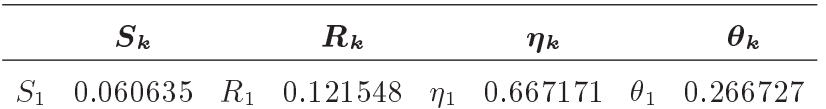

$$
\begin{aligned}
& \begin{array}{llllllll}
S_{2} & 0.094363 & R_{2} & 0.119165 & \eta_{2} & 0.558076 & \theta_{2} & 0.223112
\end{array} \\
& \begin{array}{llllllll}
S_{3} & 0.068302 & R_{3} & 0.123340 & \eta_{3} & 0.643594 & \theta_{3} & 0.257301
\end{array} \\
& \begin{array}{lllllllll}
S_{4} & 0.065122 & R_{4} & 0.112075 & \eta_{4} & 0.632486 & \theta_{4} & 0.252860 \\
\hline
\end{array} \\
& I_{11}^{*}=\left[\frac{1}{4} \sum_{k=1}^{4} \mu_{11}^{L k}, \frac{1}{4} \sum_{k=1}^{4} \mu_{11}^{U k}\right]=\left[\frac{1}{4}(0.099908\right. \\
& +0.099908+0.139872+0.159854) \\
& \left.\frac{1}{4}(0.119891+0.119891+0.159854+0.179836)\right] \\
& =[0.124886,0.14486773] \text {, } \\
& I_{11}^{-}=\left[\min _{k}\left\{\mu_{11}^{L k}\right\}, \max _{k}\left\{\mu_{11}^{U k}\right\}\right]=[\min (0.099908, \\
& 0.099908,0.139872,0.159854), \max (0.119891 \text {, } \\
& 0.119891,0.159854,0.179836)] \\
& =[0.099908,0.179836] \text {, } \\
& \eta_{1}=\frac{R_{1}}{R_{1}+S_{1}}=\frac{0.121548}{0.121548+0.060635}=0.667171,(52) \\
& \theta_{1}=\frac{\eta_{1}}{\sum_{k=1}^{4} \eta_{k}} \\
& =\frac{0.667171}{0.667171+0.558076+0.643594+0.632486} \\
& =0.266727 \text {. }
\end{aligned}
$$

The separation measures are determined by computing the distance between the normalized IVHF-decision matrix, IVHF-PIS, and IVHF-NIS by Eqs. (39)-(41).
Table 10. Separation measure.

\begin{tabular}{ccccc}
\hline \multicolumn{5}{c}{$\boldsymbol{d}_{\boldsymbol{k}}^{*}$} \\
\hline$A_{i}$ & $d_{1}^{*}$ & $d_{2}^{*}$ & $d_{3}^{*}$ & $d_{4}^{*}$ \\
\cline { 2 - 5 }$R_{1}$ & 0.021948 & 0.051061 & 0.01993 & 0.02856 \\
$R_{2}$ & 0.018890 & 0.016018 & 0.02280 & 0.03596 \\
$R_{3}$ & 0.024194 & 0.038157 & 0.01386 & 0.01290 \\
\hline \multicolumn{5}{c}{$\boldsymbol{d}_{\boldsymbol{k}}^{-}$} \\
\hline$A_{i}$ & $d_{1}^{-}$ & $d_{2}^{-}$ & $d_{3}^{-}$ & $d_{4}^{-}$ \\
\cline { 2 - 5 }$R_{1}$ & 0.047248 & 0.006819 & 0.04687 & 0.03707 \\
$R_{2}$ & 0.029835 & 0.024739 & 0.02419 & 0.01022 \\
$R_{3}$ & 0.032820 & 0.009533 & 0.04113 & 0.04117 \\
\hline
\end{tabular}

Then, the proposed collective indexes for each potential alternative are calculated by Eqs. (41)-(44); the computation process is obtained by Eqs. (54)(56). The computational results are demonstrated in Tables 10 and 11. Also, we consider a comparative analysis in Table 11 for the selection problem. In this regard, Ashtiani et al. [27] proposed a preference order ranking of interval-valued fuzzy TOPSIS (IVFTOPSIS) method, and we have utilized the IVFTOPSIS method for the purpose of ranking the potential alternatives (i.e., robots). The results of the proposed IVHF-DBGD model and IVF-TOPSIS are observed as the same. As a result, the suitable robot for performing the material handling tasks is the third candidate robot as an alternative as shown in Box VII.

\section{Conclusions}

Hesitant fuzzy group decision-making analysis is a process that provides an efficient framework for choosing and ranking the best possible alternatives respecting the conflicting criteria in the industrial selection problems under hesitant situations. In this paper, a novel distance-based group decision model; namely IVHFDBGD, was presented to solve the MCGDM problems in the Interval-Valued Hesitant Fuzzy (IVHF) environment. In the presented model, the relative importance of each criterion, as well as the preference rating values of potential alternative by regarding the selected criteria was defined in linguistic variables; then, the 


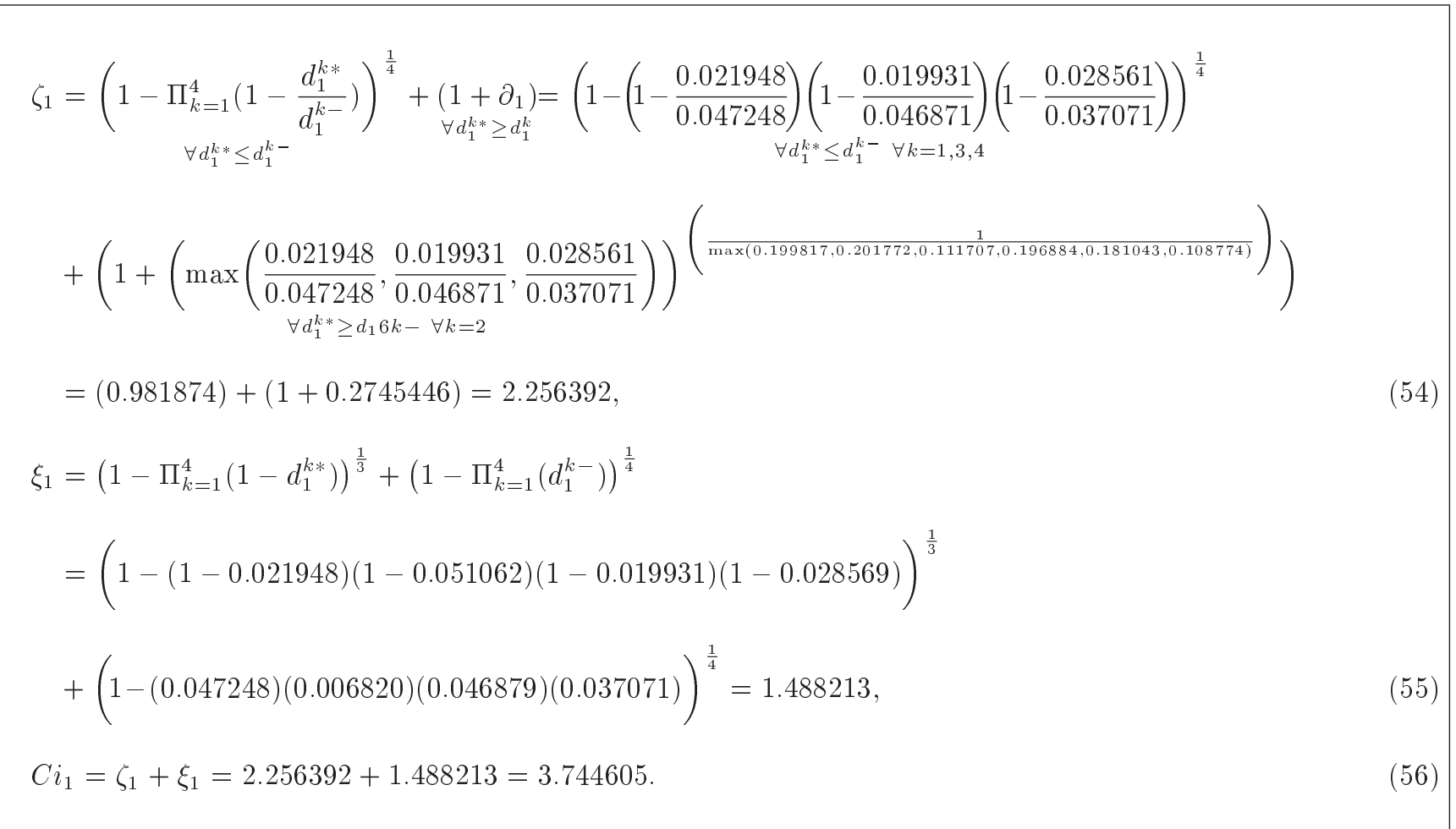

Box VII

Table 11. The proposed collective index and comparetive analysis.

\begin{tabular}{cccccc}
\hline $\boldsymbol{A}_{\boldsymbol{i}}$ & $\boldsymbol{\zeta}_{\boldsymbol{i}}$ & $\boldsymbol{\xi}_{\boldsymbol{i}}$ & $\boldsymbol{C I}_{\boldsymbol{i}}$ & $\begin{array}{c}\text { Ranked by } \\
\text { the proposed } \\
\text { IVHF-DBGD } \\
\text { model }\end{array}$ & $\begin{array}{c}\text { Ranked by } \\
\text { Ashtiani } \\
\text { et al. [27] } \\
\text { method }\end{array}$ \\
\hline$R_{1}$ & 2.256392 & 1.488213 & 3.744605 & $R_{3}$ & $R_{3}$ \\
$R_{2}$ & 2.758563 & 1.449052 & 4.207615 & $R_{1}$ & $R_{1}$ \\
$R_{3}$ & 2.189279 & 1.442066 & 3.631344 & $R_{2}$ & $R_{2}$ \\
\hline
\end{tabular}

linguistic variables were transformed to IVHFEs. Also, the weight of each criterion and Decision Makers (DMs) were considered in the process of proposed IVHFDBGD model. In this respect, the weight of each criterion was obtained by an interval-valued hesitant fuzzy entropy method and by considering the DMs' opinions about the criteria's weights. Further, a new interval-valued hesitant fuzzy order preference method by the concept of the relative closeness was presented for the weight of each DM through the group decisionmaking process. In addition, we have introduced a new collective index for discriminating among potential alternatives in the evaluation and selection processes under hesitant situations. Therefore, a practical example was presented for the robot selection in the industrial environment, indicating the suitability and validity of the proposed IVHF-DBGD model. Finally, a comparative analysis between the presented decision model and the recent IVF-TOPSIS method from the related literature was done that showed the same results. Although the proposed IVHF-DBGD model was applied for the industrial decisions, it could be used to make the most suitable decision for any areas in management and engineering problems. As a direction for future studies, the proposed IVHF-DBGD model can be enhanced by considering the Decision Support System (DSS) to decrease the needed effort and time for computations.

\section{References}

1. Mousavi, S.M., Jolai, F. and Tavakkoli-Moghaddam, R. "A fuzzy stochastic multi-attribute group decisionmaking approach for selection problems", Group Decision and Negotiation, 22(2), pp. 207-233 (2013).

2. Mousavi, S.M., Jolai, F., Tavakkoli-Moghaddam, R. and Vahdani, B. "A fuzzy grey model based on the compromise ranking for multi-criteria group decision 
making problems in manufacturing systems", Journal of Intelligent \& Fuzzy Systems, 24(4), pp. 819-827 (2013).

3. Ebrahimnejad, S., Mousavi, S.M. and Seyrafianpour, H. "Risk identification and assessment for buildoperate-transfer projects: A fuzzy multi attribute decision making model", Expert Systems with Applications, 37(1), pp. 575-586 (2010).

4. Vahdani, B., Mousavi, S.M., Hashemi, H., Mousakhani, M. and Tavakkoli-Moghaddam, R. "A new compromise solution method for fuzzy group decision-making problems with an application to the contractor selection", Engineering Applications of Artificial Intelligence, 26(2), pp. 779-788 (2013).

5. Ze-shui, X. "A multi-attribute group decision making method based on term indices in linguistic evaluation scales [J]", Journal of Systems Engineering, 20(1), pp. 8-84 (2005).

6. Makui, A., Mojtahedi, S.M. and Mousavi, S.M. "Project risk identification and analysis based on group decision making methodology in a fuzzy environment", International Journal of Management Science and Engineering Management, 5(2), pp. 108-118 (2010).

7. Yue, Z. "A method for group decision-making based on determining weights of decision makers using TOPSIS", Applied Mathematical Modelling, 35(4), pp. 1926-1936 (2011).

8. Yu, L. and Lai, K.K. "A distance-based group decisionmaking methodology for multi-person multi-criteria emergency decision support", Decision Support Systems, 51(2), pp. 307-315 (2011).

9. Xu, Z. "A method based on distance measure for interval-valued intuitionistic fuzzy group decision making", Information Sciences, 180(1), pp. 181-190 (2010).

10. Chen, T.-Y. "A signed-distance-based approach to importance assessment and multi-criteria group decision analysis based on interval type-2 fuzzy set", Knowledge and Information Systems, 35(1), pp. 193-231 (2013).

11. Zhang, H.-M. and Xu, Z.-S. "Uncertain linguistic information based C-OWA and C-OWG operators and their applications", Journal of PLA University of Science and Technology (Natural Science), 6(6), pp. 604-608 (2005).

12. Rao, R.V., Decision Making in the Manufacturing Environment: Using Graph Theory and Fuzzy Multiple Attribute Decision Making Methods, Springer, ISBN 978-1-4471-4375-8 (2007).

13. Agrawal, V., Kohli, V. and Gupta, S. "Computer aided robot selection: The "multiple attribute decision making' approach", International Journal of Production Research, 29(8), pp. 1629-1644 (1991).

14. Goh, C.-H., Tung, Y.-C.A. and Cheng, C.-H. "A revised weighted sum decision model for robot selection", Computers \& Industrial Engineering, 30(2), pp. 193199 (1996).
15. Parkan, C. and Wu, M.-L. "Decision-making and performance measurement models with applications to robot selection", Computers \& Industrial Engineering, 36(3), pp. 503-523 (1999).

16. Chu, T.-C. and Lin, Y.-C. "A fuzzy TOPSIS method for robot selection", International Journal of Advanced Manufacturing Technology, 21(4), pp. 284-290 (2003).

17. Bhattacharya, A., Sarkar, B. and Mukherjee, S.K. "Integrating AHP with QFD for robot selection under requirement perspective", International Journal of Production Research, 43(17), pp. 3671-3685 (2005).

18. Kahraman, C., Çevik, S., Ates, N.Y. and Gülbay, M. "Fuzzy multi-criteria evaluation of industrial robotic systems", Computers \& Industrial Engineering, 52(4), pp. 414-433 (2007).

19. Kumar, R. and Garg, R. "Optimal selection of robots by using distance based approach method", Robotics and Computer-Integrated Manufacturing, 26(5), pp. 500-506 (2010).

20. Kentli, A. and Kar, A. "A satisfaction function and distance measure based multi-criteria robot selection procedure", International Journal of Production Research, 49(19), pp. 5821-5832 (2011).

21. Chen, N., Xu, Z. and Xia, M. "Interval-valued hesitant preference relations and their applications to group decision making", Knowledge-Based Systems, 37, pp. 528-540 (2013).

22. Liao, H. and $\mathrm{Xu}, \mathrm{Z}$. "Subtraction and division operations over hesitant fuzzy sets", Journal of Intelligent and Fuzzy Systems, 27, pp. 65-72 (2013).

23. Zhang, Z., Wang, C., Tian, D. and Li, K. "Induced generalized hesitant fuzzy operators and their application to multiple attribute group decision making", Computers \& Industrial Engineering, 67, pp. 116-138 (2014).

24. Liang, G.-S. and Wang, M.-J.J. "A fuzzy multicriteria decision-making approach for robot selection", Robotics and Computer-Integrated Manufacturing, 10(4), pp. 267-274 (1993).

25. Vahdani, B., Mousavi, S.M., Tavakkoli-Moghaddam, R. and Hashemi, H. "A new design of the elimination and choice translating reality method for multi-criteria group decision-making in an intuitionistic fuzzy environment", Applied Mathematical Modelling, 37(4), pp. 1781-1799 (2013).

26. Torra, V. "Hesitant fuzzy sets", International Journal of Intelligent Systems, 25(6), pp. 529-539 (2010).

27. Ashtiani, B., Haghighirad, F. and Makui, A. "Extension of fuzzy TOPSIS method based on interval-valued fuzzy sets", Applied Soft Computing, 9(2), pp. 457-461 (2009).

\section{Biographies}

Hossein Gitinavard is currently an MSc student at the School of Industrial Engineering, Iran University of 
Science and Technology, Tehran, Iran. He received his BSc degree from the School of Industrial Engineering, University of Tehran, Iran, in 2013. His main research interests include fuzzy sets theory, multi-criteria decision-making under uncertainty, supply chain management and applied operations research. He has published several papers in reputable journals and international conference proceedings.

Seyed Meysam Mousavi is an Assistant Professor at the Department of Industrial Engineering, Faculty of Engineering, Shahed University, Tehran, Iran. He received a $\mathrm{PhD}$ degree from the School of Industrial Engineering at University of Tehran, Iran, and is currently a member of Iran's National Elite Foundation. He is now the head of Industrial Engineering Department at Shahed University and a member of the Iranian Operational Research Association. His main research interests include cross-docking systems planning, quantitative methods in project management, engineering optimization under uncertainty, logistics planning and scheduling, multiple criteria decision making under uncertainty, and applied soft computing. He has published many papers and book chapters in reputable journals and international conference proceedings.

Behnam Vahdani is an Assistant Professor at Faculty of Industrial and Mechanical Engineering, Qazvin Branch, Islamic Azad University, in Iran, and is a member of Iran's National Elite Foundation. His current research interests include supply chain network design, facility location and design, multi-criteria decision making, uncertain programming, artificial neural networks, meta-heuristics algorithms, and operations research applications. He has published numerous papers and book chapters in the aforementioned areas.

Ali Siadat is a Professor at Laboratoire de Conception, Fabrication Commande, Arts et Métier Paris Tech, Centre de Metz in Metz, France. His current research interests include: computer aided manufacturing, modeling and optimization of manufacturing processes, decision making in manufacturing, inspection planning, and operations research applications. He has published numerous papers and book chapters in the aforementioned fields. 